\title{
Olanzapine in practice
}

\author{
David Taylor, Siobhan Drummond and Joanne Pendlebury
}

\begin{abstract}
Prescription charts including a prescription for olanzapine were evaluated for 202 patients from 15 National Health Service trusts. In patients prescribed olanzapine for less than six weeks, the mean dose was found to be $12.4 \mathrm{mg} /$ day but $56 \%$ of patients were prescribed olanzapine as the sole antipsychotic. For patients prescribed olanzapine for longer than six weeks (when dose titration and drug crossovers were assumed to be complete) the mean dose of olanzapine was $15.8 \mathrm{mg} /$ day and $64 \%$ received olanzapine as the sole antipsychotic. A substantial proportion of patients were prescribed concurrent anticholinergic drugs (but relatively fow when on olanzapine alone). Olanzapine was used in doses higher than the standard dose recommended by the manufacturers, often in combination with typical antipsychotics. Such practices increase costs and, at least in the latter case, very probably adversely affect outcome. Studies are needed to evaluate the retative effectiveness of commonly used antipsychotic regimens in naturalistic settings. In the absence of these, olanzapine should be used as the sole antipsychotic and patients should be property assessed on lower doses before dose increases are undertaken.
\end{abstract}

Olanzapine is an atypical antipsychotic drug which has been demonstrated to be superior to haloperidol in alleviating the negative symptoms of schizophrenia (Tollefson et al, 1997a). The incidence of associated extrapyramidal sideeffects has also been shown to be lower than that of haloperidol and comparable to that of placebo (Beasley et al, 1996a). All clinical data pertaining to the superior efficacy and favourable side-effect profile of olanzapine are based on its use as monotherapy.

The recommended starting dose and routine maintenance dose of olanzapine is $10 \mathrm{mg}$ daily, taken as a single dose. Any increase to a dose greater than the routine therapeutic dose is only recommended after clinical reassessment of the patient following an appropriate trial of treatment at the routine dose.

We had observed informally that olanzapine was frequently prescribed at doses above the recommended routine therapeutic dose and with concurrent use of other antipsychotic and anticholinergic drugs. We therefore sought more formally to discover the doses of olanzapine being used in clinical practice, and to quantify the extent of concurrent prescribing of other antipsychotics and anticholinergics.

\section{The study}

Data were collected by pharmacists working in 15 trusts in south-east England. Information on in-patients, out-patients and those being discharged was collected during the week beginning 12 May 1997.

All prescription charts including a prescription for olanzapine were screened. Olanzapine dose, duration of therapy and other drugs co-prescribed were recorded. Information on patients' age and gender was obtained from prescription charts or from patient notes. 'When required' (p.r.n.) medication was also recorded.

For analysis, subjects were divided into two groups: those taking olanzapine for less than six weeks and those taking the drug for more than six weeks.

\section{Findings}

The number of patients on olanzapine in the 15 trusts was 202 ( 114 male, 88 female). The mean age was 40.5 years (s.d. 20.1), with a range of 20-90 years. Seven patients were aged over 70 years (see Table 1).

\section{Comment}

We divided subjects into two groups according to duration of therapy. Those taking olanzapine for less than six weeks we assumed to be in a titration phase of therapy where dose is adjusted according to response and tolerability. We assumed those taking olanzapine for longer than six weeks to be on maintenance doses following appropriate titration. We also assumed that previously prescribed antipsychotics would be withdrawn during the first six weeks of therapy.

Our findings demonstrate that olanzapine is used in high doses often with concurrent antipsychotics, although concurrent prescribing is less commonly observed after six weeks of therapy. These practices go against current recommendations already stated. In addition. all published clinical trials (Beasley et al, 1996a.b: Tollefson et al, 1997a,b) are based on the use of olanzapine as monotherapy and we are 
Table 1. Summary of findings

\begin{tabular}{lll}
\hline & Patients prescribed olanzapine for \\
\cline { 2 - 3 } & Less than six weeks (n=82) & More than six weeks (n=120) \\
\hline $\begin{array}{ll}\text { Dose details (mg/day) } \\
\text { Dose range }\end{array}$ & $5-20$ & $5-30$ \\
$\begin{array}{l}\text { Median dose } \\
\text { Mean dose }\end{array}$ & 10 & 15 \\
Concurrent therapy (\%) & 12.4 & 15.8 \\
On olanzapine as sole antipsychotic & $46(56)$ & $77(64)$ \\
$\begin{array}{l}\text { On olanzapine as sole antipsychotic and } \\
\text { regular anticholinergic }\end{array}$ & $4^{\prime}$ & $4^{2}$ \\
Prescribed antipsychotic when required & $12(15)$ & $23(19)$ \\
Prescribed regular antipsychotic & $24(29)$ & $20(17)$ \\
Prescribed regular anticholinergic & $16(19)$ & $18(15)$ \\
\hline
\end{tabular}

1. Nine per cent of patients on olanzapine alone.

2. Five per cent of patients on olanzapine alone.

not aware of any trials evaluating the use of olanzapine alongside other antipsychotics.

Evidently, dosing recommendations for olanzapine are not always followed in practice, thereby, in theory at least, compromising the cost effectiveness of its use. Also, the concurrent use of neuroleptics may induce extrapyramidal side-effects (as indicated by the relative high use of anticholinergics in those taking typical drugs at the same time as olanzapine) and may also negate any beneficial effect olanzapine makes on the negative symptoms of schizophrenia (Beasley et al. 1996a). Moreover, anticholinergics cause additional side-effects and may worsen or unmask tardive dyskinesias. Indeed, olanzapine shows a relatively low incidence of tardive dyskinesia (Tollefson et al, 1997b) which could be adversely affected by the co-administration of typical drugs.

From this audit it could be postulated that prescribers are unaware both of the official dosing recommendations and of the findings of currently available clinical studies. Prescribers may also be unaware of what constitutes an appropriate duration of treatment before increasing the dose. However, both these suggestions seem unlikely and our findings may indicate that olanzapine is not found to be wholly effective in clinical use at low doses and that higher doses or extra antipsychotics are then required. Clinical practice often differs from the controlled environment of a randomised clinical trial. In addition, olanzapine was perhaps being prescribed for treatment-refractory schizophrenia (a common, if informal, observation in our trusts). While olanzapine has a broadly similar pharmacological profile to clozapine there is currently no evidence to support a role in the treatment of refractory illness.
Work is currently underway in order to investigate any role for olanzapine in refractory illness, but clozapine remains the drug of choice. Double-blind clinical trials are also required to demonstrate any possible benefits of using olanzapine in combination with other antipsychotics. Until such objective evidence becomes available olanzapine should ideally be used as monotherapy. Regular review of prescriptions is beneficial in ensuring that prescribing of concurrent anticholinergics and antipsychotics is minimised where possible. Of note in our audit was the wide variation in coprescription, some centres had no patients on multiple antipsychotics, in others all patients were taking olanzapine and at least one other antipsychotic.

We have found that olanzapine is frequently prescribed at doses larger than the routine recommended dose and that it is likely to be prescribed concurrently with typical antipsychotics and anticholinergics. This may be due to lack of awareness of datasheet recommendations and findings from clinical trials, or it may be a result of the different environments of clinical trials and clinical practice. It may also be that olanzapine is being prescribed for treatment-refractory illness (for which there is no evidence to support its effectiveness), and where higher doses and concurrent antipsychotics are perhaps more likely to be used.

Olanzapine is an expensive but effective antipsychotic which has been shown to have lower incidence of extrapyramidal side-effects and to be superior to haloperidol in alleviating negative symptoms. Olanzapine ideally should not be used for refractory illness until good clinical evidence becomes available and should be prescribed as monotherapy at recommended doses in order to gain maximum benefits and to 
obtain best value for money. Naturalistic trials are needed to address the problem of different approaches to treatment seen in clinical trials and clinical practice.

\section{References}

Beasley, C. M., Tollefson, G. D.. Tran, P. V., et al (1996a) Olanzapine versus placebo and haloperidol. Acute phase results of the North American double-blind olanzapine trial. Neuropsychopharmacology. 14. 111-123.

- SANGer, T., SATTERleE, W., et al (1996b) Olanzapine versus placebo: results of a double-blind. fixed-dose olanzapine trial. Psychopharmacology. 124, 159-167.

Tollefson, G. D.. Beasley, C. M., TRAN, P. V.. et al (1997a) Olanzapine versus haloperidol in the treatment of schizophrenia and schizoaffective and schizophreniform disorders: results of an international collaborative trial. American Journal of Psychiatry. 164. 457-465.

-, - TAMURA, R. N., et al (1997b) Blind, controlled, longterm study of the comparative incidence of treatmentemergent tardive dyskinesia with olanzapine or haloperidol. American Journal of Psychiatry. 154. 1248-1254.

*David Taylor, Chief Pharmacist, Maudsley Hospital, Denmark Hill, London SE5 8AZ; Siobhan Drummond. Pharmacist, Chelsea and Westminster Hospital, London; and Joanne Pendlebury, Pharmacist, Charing Cross Hospital, London

*Correspondence

\title{
Attendance at child psychiatry clinics
}

\section{Comparison with attendance at child medical and surgical clinics}

\author{
Selim M. El-Badri and Paul McArdle
}

\begin{abstract}
A widespread perception exists that attendance at child psychiatry clinics is especially poor. The reported rate of non-aftendance at a child psychiatiy outpatient clinic is $61 \%$. However, other child health clinics also suffer a high rate of non-attendance. In this paper we examine the hypothesis that rates of non-attendance are higher in child psychiatry than in other child health out-patient clinics.
\end{abstract}

Previous studies have suggested that non-attendance and poor clinic compliance is a common problem in child health services and children clinics (Cooper \& Lynch, 1979; Novick et al, 1981; Gould et al, 1985; Potter \& Darwish. 1996). Some authors have suggested that there may be special factors influencing attendance at child psychiatric clinics. These include parental expectations of treatment offered (Plunkett, 1984), disadvantageous family structure, and the characteristics of presenting problems, especially those with antisocial behaviour (Garralda \& Bailey, 1988). Non-attendance may create inefficiencies for mental health care providers and suffering for children, which may have effects on patients' care and cost-effectiveness. This study therefore investigates whether attendance at child psychiatry clinics is poorer than at other child health clinics.

\section{The study}

Two large city centre general practices known to refer to child psychiatry were approached and their collaboration agreed. These provide services for approximately 25000 Newcastle residents. Data pertaining to patients referred from the two general practitioner practices to (a) a child and adolescent psychiatry unit, (b) ear, nose and throat (ENT) clinic and (c) a general paediatric clinic were obtained. Hospital case 